\title{
Experiencia de aula en la Fundación Universitaria Comfenalco Santander, aplicando el modelo ABP y el juego de roles como recurso pedagógico ${ }^{1}$
}

\author{
Classroom experience at the Fundación Universitaria Comfenalco \\ Santander, applying the PBL model and role play as a pedagogical
}

resource.

\section{Daniel Hernando Corzo Arévalo ${ }^{1}$}

${ }^{1}$ Docente investigador tiempo completo Fundación Universitaria Comfenalco Santander, Bucaramanga, Colombia. danielcorzoarevalo@gmail.com

Recibido: 15/feb/2019 Revisado: 30/abr/2019

Aceptado: 30/may/2019 Publicado: 30/jul/2019

\begin{abstract}
Resumen: Este artículo recopila y presenta una experiencia de aula basada en una simulación para la elaboración de políticas públicas a nivel de educación superior, empleando el Modelo de Aprendizaje Basado en Problemas -ABP y la estrategia de juego de roles como recurso pedagógico. En la simulación del juego de rol, los estudiantes fueron divididos como actores interesados en una política pública específica (Política de Vivienda) y el docente asumió la función de gobierno. Este ejercicio de simulación se desarrolló en dos grupos de diferente nivel (segundo y cuarto semestre) y tuvo como objetivo la reflexión y el entendimiento de los estudiantes sobre las variables políticas que inciden en ese proceso, destacando especialmente la existencia de un conjunto de actores políticos y sociales con intereses diferenciados y de un gobierno que -además de actuar como negociador y árbitro del proceso- cuenta también con sus propias preferencias. Apuntando a ser una herramienta pedagógica capaz de generar un aprendizaje significativo en los estudiantes y generar mayor comprensión de la complejidad inherente al ejercicio del policy making ${ }^{2}$ que pretenda generar políticas públicas legitimadas y eficaces en un contexto como el colombiano.
\end{abstract}

Palabras claves Aprendizaje basado en problemas; políticas públicas; juego de roles.

Abstract: This article presents a classroom experience based on a simulation for the elaboration of public policies at the level of higher education, using the Problem-Based Learning Model -PBL and the role-playing strategy as a pedagogical resource. In the simulation of the role play, the students were divided as interested actors in a specific public policy (Housing Policy) and the teacher assumed the role of government. This simulation exercise was developed in two groups of different levels (second and fourth semester) and had as objective the reflection and understanding of the students on the political variables that affect this process, highlighting especially the existence of a set of political actors and social with differentiated interests and a government that - in addition to acting as a negotiator and arbitrator of the process also has its own preferences. Aiming to be a pedagogical tool capable of generating significant learning in students and generate greater understanding of the complexity inherent in the exercise of policy making that seeks to generate legitimate and effective public policies in a context such as Colombia.

Keywords Problem Based Learning; Public Policy; Role Play.

\footnotetext{
${ }^{1}$ Artículo de reflexión, resultado de un ejercicio práctico durante el proceso catedrático en el módulo de Políticas Publicas y, Gobierno y territorio durante el año 2018, en la Fundación Universitaria Comfenalco Santander.

${ }^{2}$ El policy making es un proceso de aproximaciones sucesivas a un objetivo deseado, donde incluso éste se halla sometido a continuas reconsideraciones. Lindblom (1959, p. 86).
} 


\section{Introducción}

Frecuentemente, la enseñanza de los procesos de toma de decisiones en las políticas públicas, en los primeros ciclos del nivel universitario de la educación superior, se enfrenta a la dificultad de trasmitir a los estudiantes la complejidad de las racionalidades, valores e intereses involucrados en este proceso de toma de decisiones. En cambio, esos mismos alumnos acostumbran percibir al proceso de decisión, bien como una operación básicamente técnica de elección de una política que contribuirá a la obtención del bienestar general, o en el otro extremo, como una simple y directa traducción de las orientaciones ideológicas y programáticas de los gobiernos de turno.

Para superar esas visiones simplistas, hemos realizado la experiencia de colocar a grupos de dichos estudiantes, segundo semestre (Políticas públicas) y cuarto semestre (Gobierno y territorio) en el rol de varios de los actores protagónicos de las políticas públicas: integrantes del partido de gobierno o de oposición, de la alta burocracia, de gremios empresariales, de sindicatos de trabajadores o de consultores contratados. ¿Cómo razonarían y actuarían ellos mismos entonces, en un armado participativo y negociado de una política que les afecta? ¿Priorizarían el interés público o sus intereses particulares? ¿Apelarían a argumentos de naturaleza técnica o a los valores involucrados para defender sus posiciones y pretender persuadir a los otros grupos de su legitimidad y conveniencia? ¿Se involucrarían en negociaciones definidas por la afinidad ideológica de sus contrapartes, o actuarían pragmáticamente? Se ha apostado entonces, a que mediante un proceso intelectual de naturaleza inductiva se comprenda mejor la compleja racionalidad de la elaboración de las políticas públicas, a partir de las propias vivencias en un ejercicio de simulación.

En consecuencia con estas intenciones, este artículo analiza la puesta en práctica y desarrollo de esa experiencia de enseñanza-aprendizaje, tras presentar sumariamente algunas bases didácticas, metodológicas y teóricas-conceptuales que la fundamentan.

\section{El modelo de Aprendizaje Basado en Problemas - ABP, como estrategia de enseñanza-aprendizaje de las políticas públicas}

El Modelo de Aprendizaje Basado en Problemas ABP, propuesto por Barrows (1986) es un método de aprendizaje basado en el constructivismo, que consiste en utilizar problemas para adquirir conocimientos. Se caracteriza tanto por garantizar la adquisición de conocimientos, como por facilitar la consecución de habilidades y el desarrollo de actitudes importantes para el aprendizaje, como la evaluación crítica, la responsabilidad o las relaciones interpersonales. EI ABP tiene sus raíces en dos paradigmas de aprendizaje contextualizado, el aprendizaje cognitivo y la instrucción anclada, que según Morales Bueno y Landa Fitzgerald (2004), destacan la importancia de contextualizar la enseñanza en problemas reales que plantea el mundo profesional. Este método hace posible el aprendizaje de principios aplicables a diversas problemáticas, mediante la exploración de problemas muy diferentes (Branda, 2001).

En este sentido, plantear problemas desde la enseñanza de las políticas públicas permitirá al docente promover el desarrollo de una cultura de trabajo colaborativo, involucrar a todos los miembros del grupo en el proceso de aprendizaje, promover habilidades interpersonales, propicia la participación entre los estudiantes, generando que desempeñen diferentes roles en las labores propias de las actividades diseñadas, que les permitirán ir adquiriendo los conocimientos necesarios para enfrentarse al problema retador.

El Aprendizaje basado en Problemas - ABP se sustenta sobre el principio de utilizar los problemas como punto de partida para la adquisición e integración de nuevos conocimientos (Barrows, 1986). El desarrollo de este método requiere: compromiso del estudiante en su aprendizaje, formación de pequeños grupos, el papel de guía y consultor del aprendizaje del docente, la organización del currículo sobre los problemas que estimulan el aprendizaje y que 
desarrollan las habilidades en la resolución de problemas (Barrows, 1996).

El modelo ABP promueve el conocimiento aplicado a escenarios sociales reales, es por ello que podemos precisar el análisis a todos los elementos que conllevan las políticas públicas, desde su concepción hasta su continua implementación, seguimiento y evaluación. Esto significa que las propuestas refieren al estudio de casos precisos con una solución a los problemas propuestos.

Ahora bien, ¿Qué factores abonarían la conveniencia de empleo de este modelo de aprendizaje? Luego de pasar revista a los aportes de varios académicos que abordaron el tópico encontramos que:

- Incrementa significativamente el uso de estrategias para resolver problemas y en la obtención de información (Stepien, 1993).

- Mejora el conocimiento del estudiante sobre la materia, la habilidad para resolver problemas del mundo real y su motivación para el aprendizaje (Nendaz y Tekian, 1999)

- Genera un mayor desarrollo de competencias profesionales (Koh et al., 2008).

- Fomenta el pensamiento crítico, la compresión, a aprender a aprender y el trabajo cooperativo (White, 1996).

- Aumenta la capacidad crítica para analizar la información que aparece en el proceso de búsqueda (Molina et al., 2003).

- Promueve el desarrollo de las capacidades metacognitivas de los estudiantes (Downing et al., 2009)

\section{El juego de rol como estrategia de enseñanza-aprendizaje en las políticas públicas}

La estrategia del juego de rol forma parte de uno de los recursos ampliamente empleados en la formación de estudiantes de diferentes áreas de las ciencias sociales, siendo las políticas públicas parte de las ciencias sociales, más específicamente de las ciencias políticas. Existen aplicaciones del juego del rol en el campo de las políticas públicas que tienen por propósito facilitar una comprensión más profunda a los estudiantes de los conceptos, procesos y racionalidades de un campo determinado, haciéndolos trabajar y reflexionar sobre la representación de un entorno real. (Assal y Blake, 2006; Baranowski, 2006; Endersby y Webber, 1995, Lay y Smarick, 2006; O'Reilly, 2008).

El juego de rol, supone por su parte, una modalidad particular de la simulación. En él los estudiantes se transforman en actores ("jugadores") que deben actuar de la manera que ellos juzguen apropiada según el rol que deben desempeñar. Si bien deben ajustarse a reglas como en toda simulación, en este caso los estudiantes gozan de un albedrio más amplio para actuar y tomar decisiones, de acuerdo a su propia interpretación y estrategia a aplicar, al escenario virtual predefinido. Por otra parte, si bien es un juego, no necesariamente es uno de suma cero; esto es, puede ambientar tanto modalidades de actuación competitivas como colaborativas.

Según Gil (2010), las características del juego de rol son las siguientes:

- Reducen una situación real a la escala del aula, haciendo su complejidad abarcable para los alumnos.

- Plantean una acción que implica y motiva a los participantes.

- Dan la posibilidad de trabajar sobre sistemas sociales complejos.

- Permiten trabajar múltiples aspectos actitudinales: tolerancia, empatía, respeto, trabajo en grupo, espíritu crítico, correcta expresión, capacidad de síntesis.

- Desarrollan la capacidad de expresión y comprensión.

- Introducen una ruptura con el ritmo habitual de las clases.

Ahora bien, ¿Qué factores abonarían la conveniencia de empleo de esta modalidad didáctica? Luego de pasar revista a los aportes de varios académicos que abordaron el tópico, Sutcliffe (2011) identifica los siguientes: 
- Impulsar a los estudiantes a reflexionar sobre sus conocimientos y a extraer conjuntamente conclusiones sobre los temas planteados en el curso.

- Favorecer una mejor apreciación de las características de los distintos roles y de sus responsabilidades, desarrollando la empatía de los estudiantes con diferentes posturas y sentimientos más allá de sus propias asunciones y expectativas.

- Contribuir a una mejor dinámica grupal, favoreciendo la interactividad.

- Otorgar "vida" a conceptos teóricos abstractos, colocándolos en situaciones que les resultan familiares a los intérpretes.

- Contribuir al desarrollo de destrezas clave, en términos de habilidades comunicativas y sociales.

\section{Algunas premisas teórico - conceptuales para el análisis de las políticas públicas}

Naturalmente, la vinculación de esta simulación a los dos módulos a nuestro cargo, requirió la explicación de ciertos desarrollos de la teoría de las políticas públicas en algunas sesiones de clases previas. Entre esos conceptos se hallaban:

- La concepción pluralista de la política pública como un "juego de poder", en el que participan múltiples actores con valores, intereses, estrategias y recursos diferentes (Lindblom, 1991), si bien corregida asignando un mayor protagonismo al gobierno que el que le atribuye esta corriente teórica, según sugieren otras indagaciones (Grindle y Thomas, 1991);

- La teoría de la elección publica, que concibe a los decisores de políticas como maximizadores de utilidades y orientados por la prosecución de intereses particulares, sean estos funcionarios (Niskanen, 1980) o partidos políticos (Downs, 1992).

- La conceptualización de que los colectivos participantes en la formulación de las políticas, desarrollan determinadas estrategias (Lindblom,
1991) en las que movilizan recursos de poder específicos a su disposición (Subirats et al., 2008).

- La existencia de actores con "poder de veto" sobre alternativas decisorias que no le satisfacen en ciertas políticas sectoriales, y por ende, cuya adhesión es requerida para alterar el statuquo (Tsebelis, 2000).

- El postulado de que los partidos políticos, además de los intereses de conservación y ampliación de sus espacios de poder institucionalizado, se movilizan también de acuerdo a orientaciones ideológicas y programáticas que inciden en el direccionamiento de las políticas públicas que propugnan (Boix, 2002).

\section{Diseño de la simulación}

Las reglas en líneas generales, fueron los siguientes: Se seleccionó una política sectorial (un plan de vivienda) y a partidos políticos que en su definición guardan una relación aproximada con sus referentes reales de Colombia, a efectos de otorgarle visos de realidad. Básicamente, el caso es el siguiente: la definición de una política de vivienda destinada a los sectores de menores ingresos de la población, en la que distintos actores políticos y sociales deberán establecer sus características en algunas dimensiones fundamentales.

Estas dimensiones o ejes centrales en cuestión son los siguientes:

- Su modalidad de construcción, y el protagonismo relativo de distintos actores: ¿Se construirán bajo la dirección y con personal del aparato burocrático estatal, se confiarán a empresas privadas o contarán con un fuerte componente de voluntariado social?

- El tiempo de construcción: ¿Se apostará a soluciones habitacionales de rápida concreción, o a alternativas superiores que demorarían la respuesta a la necesidad social?

- La estrategia de financiamiento para obtener los recursos necesarios para solventar el programa: ¿Se aumentará la carga impositiva, se recortarán y 
transferirán otros rubros del gasto público o se acudirá a préstamos externos?

- La definición del tipo de vivienda a construir: ¿Cómo se balancearán los valores contrapuestos de la calidad de las viviendas, por un lado y de la cantidad a construir, dado un presupuesto acotado?

En la simulación, además del gobierno, se identificaron seis actores: el partido de gobierno, una coalición de partidos de oposición, la alta burocracia del Ministerio de Vivienda, una gremial de empresas de la construcción, el sindicato de trabajadores del sector, e intelectuales que asesoraban en la toma de decisiones. Se dividió a los estudiantes en seis grupos que desempeñaron el rol de cada uno de esos actores (asignados por su propia elección, y en su defecto, por sorteo). Para cada uno de ellos se explicitó un perfil de orientación ideológico-programática y de intereses particulares (necesariamente caricaturizados con fines didácticos), a los que se asoció una determinada estructura racional de preferencias en cuatro "ejes de política" a resolver (la modalidad de construcción de las viviendas, el plazo de ejecución, las fuentes de financiamiento, el tipo de vivienda a construir).

Se asignó a cada actor-grupo un determinado número de "votos" o "recursos" que representaban su poder de influencia en la decisión de la política, que podían distribuir de la manera que prefirieran en la incidencia (votación) de los cuatro ejes de políticas. Además, se concedió un solo derecho de "veto" a cada actor. El gobierno como tal (representado por el docente) no votaba, pero manejaba los tiempos de la negociación, disponía cuartos intermedios, fundamentaba posiciones, promovía acuerdos y disponía de dos recursos de veto, que podía usar además para levantarlo y que pudieran interponer otros actores.

La mecánica del juego contemplaba varias fases sucesivas, aplicadas para cada uno de esos cuatro ejes: articulación de intereses (definición de estrategias intra-grupos), argumentación pública a favor de su opción de política preferida, agregación de intereses (negociación intergrupal), votación plenaria, recuento de recursos/votos, y (eventualmente) aplicación de vetos a la decisión que recogió la mayoría de los votos. Cada opción vetada era descartada y se volvía a desarrollar todo el proceso, culminando en una nueva votación sobre las opciones restantes. Al final de la resolución de cada uno de los ejes de políticas, se imputaba a cada actor, determinada cantidad de "puntos" acorde con lo favorable que le resultó la decisión adoptada según su estructura de preferencias predeterminadas.

El juego culminaba cuando se decidiera sobre los cuatro ejes de políticas y en ese caso se realizaba una ordenación final de los actores según los puntos obtenidos; o bien, cuando se truncara el proceso de formulación de la política a causa de su obstrucción por sendos vetos interpuestos contra todas las opciones disponibles en cualquiera de los ejes a decidir. En este caso, la política quedaría sin efecto y todos los actores perderían.

\section{El desempeño de los grupos estudiantiles en la simulación}

La simulación se realizó en dos módulos diferentes del programa de Turismo y Desarrollo Local, el módulo políticas públicas de segundo semestre y, Gobierno y Territorio de Cuarto, ambos con resultados diferentes: en el primer caso, los grupos de estudiantes llegaron a un acuerdo inicial en la definición de los primeros ejes, sin pasar por obstrucción de la política. En el segundo, la política llego a término, luego de sobreponerse a varios intentos de obstrucción.

En ambos casos, los estudiantes encarnaron con toda fidelidad y entusiasmo la identidad de los actores que representaban, procurando en cada fundamentación, negociación y votación, maximizar sus recompensas, con independencia de cualquier reflexión sobre intereses más generales no explicitados (pero evidentes en una política de vivienda focalizada) y en ocasiones en desmedro del perfil ideológico del mismo actor (por ejemplo, el "sindicato de trabajadores de la construcción" intentó vetar toda esta política de indudable interés social, cuando sus preferencias no se vieron contempladas). Por lo común, las negociaciones entre grupos tampoco 
tuvieron como referencia su afinidad ideológica, sino simplemente intereses comunes en las votaciones. En ninguna ocasión se laudó un eje por argumentos contrastados por evidencia empírica acerca de la conveniencia de una u otra solución, sino que la fundamentación de las ventajas o desventajas de ellas, se defendió pretendiendo legitimarlas en base a presuntos intereses nacionales, que siempre coincidían con el interés de cada actor.

Un elemento clave en la simulación es que en ninguna parte de las reglas de juego se indicó que existirian "ganadores", y menos aún que estos serían aquellos que maximizaran el interés particular: la asunción de ésta supuesta pauta no explicitada fue espontanea, y orientó toda la estrategia desarrollada por los estudiantes. Ningún grupo apostó a estrategias que privilegiaran supuestos intereses generales con independencia de sus roles, procurando otra forma de satisfacción que no fuera la del interés particular.

\section{Resultados}

Una vez finalizada la simulación, se invitó a reflexionar grupalmente acerca del desarrollo de la experiencia y especialmente, de los móviles que impulsaron a la adopción de las estrategias referidas. A posteriori se les indicó la profundización del análisis, poniendo en relación los aportes teóricos volcados en otras clases del módulo y la simulación, por medio de un ensayo de carácter grupal, que tiene como pautas la movilización y contrastación de las premisas teóricoconceptuales manejadas, y de preguntas disparadoras de la reflexión, como las siguientes:

- ¿Por qué consideran que se llegaron a tomar las decisiones adoptadas?

- ¿Entienden que los distintos grupos actuaron racionalmente?

- ¿Privilegiaron con su accionar el interés general o intereses particulares?

- ¿Se negoció eficazmente para lograr un equilibrio de ambos términos? ¿Por qué algunos actores obtuvieron más beneficios ("puntos") que otros? ¿Los que obtuvieron menos beneficios, como deberían haber corregido sus prácticas para lograr un resultado positivo?

- ¿Qué conclusiones han extraído de la experiencia en cuanto a las posibilidades y condiciones deseables para obtener políticas públicas "legitimadas" y de "equidad pertinente" (Repetto, 2004)?

- ¿La práctica sugiere que convendría sacrificar parcialmente una finalidad en beneficio de otra (por ejemplo, políticas menos participativas, pero pretendidamente más eficaces)?

No obstante, tanto de la propia evaluación que los estudiantes realizan de la experiencia, como del análisis compartido luego de su realización y de las observaciones y reflexiones incluidas en los ensayos de los estudiantes, se evidencia preliminarmente una comprensión de las elaboraciones teóricas y de la sustancia y complejidad del proceso de toma de decisiones en las políticas públicas, mucho más cercana a la realidad que el que detectaban los mismos participantes al comienzo del módulo y de mayor rendimiento que el obtenido en otras versiones de cursos similares que emplearon estrategias más clásicas de enseñanza. Particularmente, los estudiantes comprendieron más cabalmente, entre otros tópicos:

- Las razones del pragmatismo que suele orientar el proceso de elaboración de las políticas públicas en sociedades democráticas y plurales, que abonan en términos generales las explicaciones incrementalista de estas hechuras;

- La complejidad de identificar un "interés general" más allá de las preferencias de los diversos grupos;

- La importancia y la necesidad de la negociación entre actores, más allá de la distancia ideológica o programática existente entre ellos;

- La dificultad para tomar decisiones basadas exclusivamente en valores, en un marco de constricciones políticas, financieras y temporales;

- Las limitaciones efectivas del conocimiento para dirimir cuestiones (y en cambio, su permanente utilización con fines partidaristas);

- La tensión existente entre hechuras de políticas más inclusivas y otras supuestamente más 
"eficaces" para el logro de objetivos que se reputan valiosos.

\section{Conclusiones}

Resultó bastante evidente que los estudiantes habían logrado una compresión más cercana a la complejidad del policy making y especialmente a su naturaleza fundamentalmente política, y desarrollado una mayor empatía con los actores políticos y sociales más relevantes. A partir de ese punto de comprensión, el cierre de la simulación hizo énfasis en dos aspectos: Por un lado, el reconocimiento de dos limitaciones fundamentales de la simulación:

- La carencia de una dimensión temporal más extendida (en la cual la confianza o desconfianza entre los actores jugaría un papel importante, y por tanto condicionaría las interacciones), y

- la reducción del juego a una sola política, mientras en la realidad los distintos issues se tramitan simultáneamente, complejizando el juego y condicionando las estrategias de los actores.

Por otro lado, es posible conjugar el relativismo inherente a varios de los asertos que emergieron de la simulación con la necesidad de construir -pese a todo- prácticas políticas que favorezcan juegos de suma positiva y prohíjen, por tanto, mejores políticas públicas. Esto es, la necesidad de desarrollar desde el reconocimiento de la multiplicidad de actores con visiones e intereses divergentes que tradicionalmente han descripto las corrientes pluralistasincrementalista, formatos $y$ experiencias que maximicen las estrategias negociadoras (Fisher y Ury, 1985) y el uso del conocimiento (Dror, 1989) para resolver problemas colectivos.

Por tanto, entendemos que esta experiencia de aplicar el Modelo de Aprendizaje Basado en Problemas con su estrategia de juego de rol -con los aprendizajes y adaptaciones que correspondan- es una alternativa digna de consideración a la hora de plantearse la enseñanza en este campo.

\section{Referencias}

Asal, Victor y Blake, Elizabeth (2006). Creating Simulations for Political Science Education. Journal of Political Science Education Vol. 2, No 1, pp. 1-18.

Baranowski, Michael (2006). Single Session Simulations: The Effectiveness of Short Congressional Simulations in Introductory American Government Classes. Journal of Political Science Education Vol. 2, No 1, pp. 33-49.

Barrell, J. (1999). El aprendizaje basado en problemas. Un enfoque investigativo. Buenos Aires: Manantial.

Barrett, T. (2005). Understanding problem-based learning. En Handbook of enquiry \& problem based learning. In T. Barrett, I. MacLabhrainm, H. Fallon (Eds.), pp 1325.

Barrows, H.S. (1986) A Taxonomy of problem- based learning methods. Medical Education, 20: 481-486.

Barrows, HS. (1996) Problem-Based learning in medicine and beyond: A brief overview. In WILKERSON L., GIJSELAERS W.H. (eds) Bringing Problem-Based Learning to Higher Education: Theory and Practice, San Francisco: Jossey-Bass Publishers, pp. 3-12.

Boix, Carles (2002). Partidos políticos, crecimiento e igualdad. Madrid: Alianza. Bolzan,

Branda, L. (2001). Aprendizaje Basado en Problemas centrado en el estudiante, orientado a la comunidad. En PULPEIR, A. (dir.) Aportes para un cambio curricular en Argentina 2001. Buenos Aires: Universidad de Buenos Aires. p. 79-101. Disponible al 5.4.2018 en http://www.psico.uniovi.es/fac_psicologia/paginas_ee es/Adaptacion_de_profesorado/metodos_docentes/a prendizaje_basado_en_problemas_aulafutura.pdf

R.F.F.A. (2003). O aprendizado na internet utilizando estrategias de roleplaying game (RPG). Tesis de doctorado, Universidade Federal de Santa Catarina, Florianopolis, Brasil.

Downing, K., Kwong, T., Chan, S. W., Lam, T. F., \& Downing, W. K. (2009). Problem-based learning and the development of metacognition, Higher Education, 57 (5), 609-621.

Downs, Anthony (1992). Teoría económica de la acción política en una democracia, en G. Almond et al. Diez Textos Básicos de Ciencia Política. Barcelona: Ariel Ciencia Política.

Dror, Yehezkel (1989). Public Policymaking reexamined. New Brunswick and Oxford: Transaction Publishers.

Endersby, James W. y David J. Webber (1995). Iron Triangle Simulation: A role-playing game for undergraduates in 
Congress, interest groups, and public policy classes. PS: Political Science and Politics, vol. 28, No3, pp. 520-523.

Ferreira Borges, Aurelio et al. (2011). Estrategias de enseñanza-aprendizaje con el apoyo del juego pedagógico roleplaying game. Revista Iberoamericana de Educación, Vol. 56, No 3.

Fisher, Roger y Ury, William (1985). Sí, de acuerdo! Como negociar sin ceder. Bogota: Norma.

Gil, Carolina (2010). "El juego de rol aplicado a la educacion ambiental". Disponible al 5.4.2018 en http://www2.fe.ccoo.es/andalucia/docu/p5sd7055.pdf

Grindle, Merilee S. y Thomas, John W. (1991). Public choices and policy change. The political economy of reforms in developing countries. Baltimore: The Johns Hopkins University Press.

Koh, G. C. H., Khoo, H. E., Wong, M. L. \& Koh, D. (2008). The effects of problem-based learning during medical school on physician competency: A systematic review. CMAJ, 178, 34-41.

Lay, Celeste J., y Kathleen J. Smarick (2006). "Simulating a Senate Office: The Impact on Student Knowledge and Attitudes". Journal of Political Science Education Vol. 2 No 2, pp. 131-146

Lindblom, Charles (1991). El proceso de elaboración de las políticas públicas. Mexico DC: Porrua. Niskanen, William (1980). Cara y cruz de la burocracia. Madrid: Espasa Calpe.

LINDBLOM, Ch.E., "The Science of Muddling Through", en Public Administration Review, vol. 19, 1959, pp. 79-89.

Molina, J. A., García, A., Pedraz, A. \& Antón, M. A. (2003). Aprendizaje basado en problemas: una alternativa al método tradicional. Revista de la Red Estatal de Docencia Universitaria, 3 (2), 79-85.

Morales Bueno, P., y Landa Fitzgerald, V. (2004). Aprendizaje Basado en Problemas. En: Theoria, $\mathrm{n}^{\circ}$ 13(1). Disponible al 5.4.2018 en http://campus. usal.es/ ofeees/NUEVAS_METODOLOGIAS/ABP/13 .pdf

Nendaz, M. R. \& Tekian, A. (1999). Assessment in problembased learning medical schools: A literature review. Teaching and Learning in Medicine, 11 (4), 232-243.

O'Reilly, Patricia (2008). "Finding the balance in public policy simulations and role playing". Paper presentado al Anual Meeting of the APSA Teaching and Learning Conference, San Jose, California.

Repetto, F. (2004). Capacidad estatal. Requisito para el mejoramiento de la política social en América Latina. Documentos de trabajo del INDES.

Restrepo, Bernardo. (2005) Aprendizaje basado en problemas $(\mathrm{ABP})$ : una innovación didáctica para la enseñanza universitaria Educación y Educadores, vol. 8, pp. 9-19 Universidad de La Sabana Cundinamarca, Colombia.

Solaz-Portolés, JJ, et al. (2011). Aprendizaje basado en problemas en la Educación Superior: una metodología necesaria en la formación del profesorado. Depart. Didàctica de les Ciències Exper. i Socials

Subirats, J. et al. (2008). Análisis y gestión de políticas públicas. Barcelona: Ariel.

Sutcliffe, Mark. Simulations, games and role - play. Disponible al $\quad 5.4 .2018$ en www.economicsnetwork.ac.uk/handbook/printable/ga mes_vs.pdf

Stepien, W. J. (1993). Problem-based learning: As authentic as it gets. Educational Leadership, 50 (7), 25-28.

Tsebelis, George (2000). Veto players and institutional analysis, en Governance, No 13, pp. 441-474.

White, H B. (1996). Dan tries problembased learning: A case study. En L. Richlin (Ed.), To improve the Academy, Vol. 15 (pp 75-91). Stillwater, OK: New Forum Press \& Professional and Organizational Network in Higher Education. 